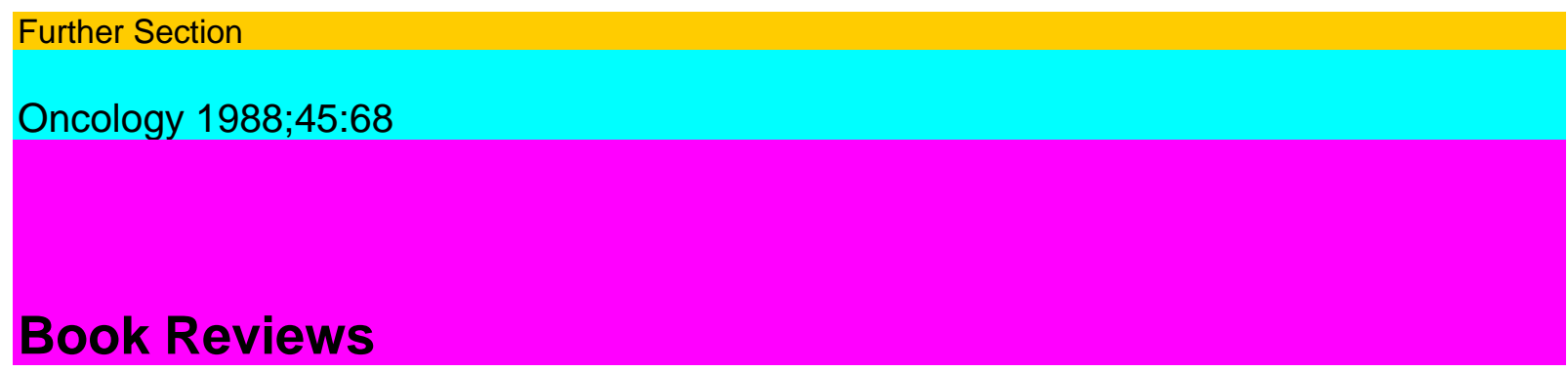

\title{
H.H. Sedlacek
}

Tumor Therapy with Tumor Cells

and Neuraminidase

Beitriige zur Onkologie, vol. 27 Karger, Basel 1987

VII + 96pp; SFr. 50.-/DM 60.-/US \$28.00 ISBN 3-8055^549-5

The monograph is devoted to the problem of cancer immunotherapy by means of inactivated tumor cells with increased immunogenicity. The book consists of 4 chapters. The first 2 chapters deal with the influence of neuraminidase on the cell membrane. Its immunomodulating effect has been highlighted. Having compared vast though contradictory literature data with the results of his own, the author came to a conclusion that neuraminidase-related activation of the immune response to antigens of both tumor and normal cells occurred due to demasking antigen determinants on the cell surface.

Chapters 3 and 4 comprise the results of experimental and clinical study of antitumor activity (vibro choleral neuraminidase) treated inactivated tumor cells. The experiments with transplanted animal tumor have shown that the antitumor effect depends not only on the number of inactivated tumor cells but also on the amount of neuraminidase used. It has also been demonstrated that joint administration of neuraminidase and inactivated tumor cells to tumorbearing animals appeared more efficient than preliminary treatment of cells with neuraminidase. The author suggests chessboard vaccination as most effective. It consists in intradermal injection of increasing numbers of tumor cells, each mixed with increasing numbers of neuraminidase. Chessboard administration of inactivated tumor cells and neuraminidase to patients with cancer of the stomach, breast, prostate and colon appeared beneficial in a number of cases. Most encouraging results have been obtained in colon cancer. Taking into account the absence of side effects, usually accompanying chemotherapy, the proposed treatment modality seems rather promising and deserves further investigations.

N.P. Napalkov, Leningrad

J. Rygaard, M. Spang-Thomsen Immune-Deficient Animals in Biomedical Research Karger, Basel 1987

XVI+ 420 pp.; SFr. 267.- DM 320.- US\$ 148.50

ISBN 3-8055^385-9

The Proceedings of the 5th International Workshop on Immune-Deficient Animals in Biomedical Research provide a good opportunity to combine all the up-to-date literature by international groups working in this area.

This text covers different aspects of biomedical research applied to immune-deficient animals, the focus being primarily on nude mice as an animal model to grow and study the biology of human tumors. Contributions include: tumor markers and biology, pattern of metastasis and therapeutic approaches. Basic immunology and immunopathology are also treated; of particular interest is the section on immunity of the Skin and mucous membrane - an area of increasing exploration. 
The book reflects the broad and heterogeneous range of interactions between immunology and oncology.

S. Garattini, Milano

Miriam C. Poirier

Carcinogenesis and Adducts in Animals and Humans

Progress in Experimental Tumor Research, vol. 31 Karger, Basel 1987

VIII+ 116 pp.; SFr. 119.-/DM 142.-/US \$ 66.25 ISBN 3-8055^1457-X

Since it was recently shown that a chemical carcinogen which induced breast carcinomas in rats activated the oncogene ras by a mutation which may be explained by a specific DNA adduct, the relationship between chemical carcinogenesis and adduct formation is of great scientific importance.

This book reports the proceedings of a workshop held in 1986. The participants are all experts in this field, but unfortunately European specialists did not participate. In ten brief chapters, illustrated excellently, highly sensitive techniques recently developed are described and new data summarized.

Multiple factors influence the analyses of DNA and protein adduct formation in experimental animals or humans exposed to chemical carcinogens by occupation, lifestyle, diet or medical treatment. Genetic heterogeneity of metabolic activation and exposure to other exogenous compounds that may alter metabolism as well as adduct formation, stability and removal represent such factors.

Several new sensitive techniques are described for detecting exceptionally small quantities of adducts without requiring test chemicals to be radioactive. These methods are based on specific monoclonal antibodies. In addition, high-resolution thin-layer chromatography of 32P-labeled nucleotides is described.

$\mathrm{T}$. This book is also a contribution to the rapidly advancing area of adduct monitoring in carcinogen-exposed human cohorts: DNA adducts associated with maternal smoking during pregnancy have been found in human placenta

others in cancer patients receiving cisplatin chemotherapy. In addition

first findings indicate that human serum contains adduct-specific antibodies. However

for final evaluation of the biological significance of protein and DNA adducts a larger data base should become available.

J. Denner

Berlin-Buch 